\title{
Finite Element Dynamics of Human Ear System Comprising Middle Ear and Cochlea in Inner Ear
}

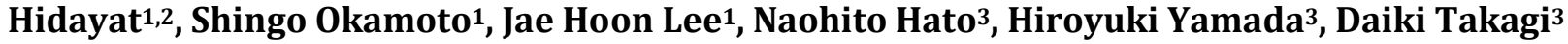 \\ ${ }^{1}$ Graduate School of Science and Engineering, Ehime University, Matsuyama, Japan \\ ${ }^{2}$ Mechanical Engineering Department, State Polytechnic of Samarinda, Samarinda, Indonesia \\ ${ }^{3}$ Department of Otorhinolaryngology, Head and Neck Surgery, Ehime University School of Medicine, Matsuyama, Japan \\ Email: b861008a@mails.cc.ehime-u.ac.jp
}

How to cite this paper: Hidayat, Okamoto, S., Lee, J.H., Hato, N., Yamada, H. and Takagi, D. (2016) Finite Element Dynamics of Human Ear System Comprising Middle Ear and Cochlea in Inner Ear. J. Biomedical Science and Engineering, 9, 597-610. http://dx.doi.org/10.4236/jbise.2016.913051

Received: October 13, 2016

Accepted: December 5, 2016

Published: December 8, 2016

Copyright $\odot 2016$ by authors and Scientific Research Publishing Inc. This work is licensed under the Creative Commons Attribution International License (CC BY 4.0).

http://creativecommons.org/licenses/by/4.0/

\begin{abstract}
A human middle ear consists of an eardrum and three ossicles which are linked by each other, and connect with the eardrum and an inner ear. The inner ear consists of a cochlea and a vestibular system. An abnormality of the human middle ear such as ossicular dislocation may cause conductive hearing loss. The conductive hearing loss is generally treated by surgery using artificial ossicles. The treatments of conductive hearing loss require a better understanding of characteristics and dynamic behaviors of the human middle ear when the sounds transmit from outer inner to inner ear. The purpose of this research is to simulate the dynamic behaviors of a human ear system comprising the middle ear and the cochlea in the inner ear using the finite element method (FEM). Firstly, the eigen-value analysis was performed to obtain the natural frequencies and vibration modes of the total ear system. Secondly, the frequency response analysis was carried out. Thirdly, the time history response analyses were performed using human voices as the external forces. In the time history response analyses, the sounds created as input sound pressures were used. Human voices, for example vowels " $P$ ", " $u$ " and " $e$ " as input sound pressures were created by using the sound pressures downloaded from the opening samples of human voices as wav files in a website. Then it was clarified that the high frequency components of sounds are reduced by the middle ear system.
\end{abstract}

\section{Keywords}

Eardrum, Middle Ear, Dynamics, Human Voice, Finite Element Method

\section{Introduction}

Hearing loss is one of the most severe problems in usual life activity of the human being. WHO reports that over $5 \%$ of the world's population have hearing loss. Conductive 
hearing loss is the most common case due to problems in the middle ear or the outer ear at times. A human middle ear which includes an eardrum and three ossicles (malleus, incus and stapes) is a mechanical system to transmit sounds from outer ear to inner ear. The conductive hearing loss occurs when sounds hardly transfer through the ear canal to the eardrum and three ossicles. The otosclerosis in which the ossicles in middle ear become stiff or the dislocation of three ossicles causes severe conductive hearing loss. Conductive hearing loss can often be treated with a surgery using artificical ossicles.

Several researchers on middle ear system use finite element analysis in their study. Finite Element Analysis (FEA) is a type of numerical analyses that uses finite element method to simulate/analyze the behaviors of mechanical structures and components under a variety conditions. The FEA is a powerful tool to analyze the vibration of a middle ear because the middle ear has a complicated shape. The major benefits of finite element analysis in this study are to calculate natural frequencies, vibration modes, frequency response and time history response analyses of a human middle ear which cannot be obtained by using conventional methods. The past researchers on middle ear system are introduced as follows. Wada et al. measured Young's modulus, thickness and damping ratio of the human eardrum by using measuring aparatus developed by themselves [1]. As for dynamics analysis on the middle ear system, a cat eardrum was firstly investigated by a finite element analysis on the curved conical eardrum by W. R. I. Funnell and C. A. Laszlo [2]. D. D. Greef et al. performed dynamics analysis on a new anatomically-accurate model composed of the tympanic membrane and malleus using the finite element method [3]. Computational modeling methodology of multi-body system was examined in order to simulate and study the middle ear mechanical response to acoustic stimuli by F. Bohnke et al. [4]. Y. Liu et al. carried out a three-dimensional finite element analysis of human ear in order to analyze lesion of ossicular chain [5]. E. Skrodzka and J. Modlawska performed modal analysis of the human tympanic membrane of middle ear using the finite element method [6]. C. F. Lee et al. proposed a practical approach that uses high-resolution computed tomography (HRCT) to derive models of the middle ear for finite element analysis [7]. R. Z. Gan et al. performed a three-dimensional finite element analysis of the human ear that included the external ear canal, eardrum, ossicular bones, middle ear suspensory ligaments/muscles, and middle ear cavity [8]. A practical and systematic method for reconstructing accurate computer and physical models of entire human middle ear were proposed by Q. Sun et al. [9]. T. Koike et al. performed the finite element analysis of the human middle ear and compared calculated results with measurement data [10]. The displacements of the tympanic membrane in a human ear were measured by using a Laser Doppler Vibration (LDV) and compared with those of a finite element analysis of the middle ear by T. S. Ahn et al. [11]. S. Ihrle et al. developed a nonlinear numerical model of the human middle ear based on the elastic multibody system (EMBS) method [12]. Middle ear responses to both air conduction and bone conduction excitations were measured at the umbo and lateral process of the malleus by Kenji Homma et al. [13]. 
Then the authors reported on a three dimensional model of human middle ear system, eigen-value analysis, frequency response analysis and time history response analysis. In the time history response analysis of the previous research, formant frequencies were used as the frequencies of the external forces. Here, the frequencies, $F_{1}=750[\mathrm{~Hz}]$, $F_{1}=1250[\mathrm{~Hz}]$ and $F_{1}=2500[\mathrm{~Hz}]$ are the first Formant frequencies of " $o$ ", " $a$ " and " $i$ " in human simple vowels respectively [14].

In the present paper, time history response analyses were carried out using human voices created as external forces. The Hyper Works (finite element analysis code) was used to carry out dynamics analyses of the total ear system. Firstly, the eigen-value analysis was carried out to obtain the natural frequencies and the vibration modes. Then, the frequency response analysis was performed to show the response of total ear system in the frequency range 100 to $10,000[\mathrm{~Hz}]$. In time history response analysis, firstly, the sound pressures were downloaded from a website opening samples of human voices as wav files for the input sound pressure. Then, the sounds as input data were created by using downloaded sound pressures. The sound pressure on three vowels " $i$ ", " $u$ " and " $e$ " as wav files were used. Finally, it was obtained that the high frequency components of sounds were reduce by the middle ear system.

\section{Finite Element Model Comprising Middle Ear and Cochlea in Inner Ear}

\subsection{Middle Ear}

Figure 1 shows the finite element model of total ear system containing a middle ear, a cochlea in an inner ear, four ligaments, a tendon and a tensor tympanic membrane. The middle ear consists of an eardrum and three ossicles, namely malleus, incus and stapes. The eardrum is a concave membrane that separates the outer ear and the middle one. The ossicles are three linked small bones that connect the eardrum to the cochlea. A sound wave transmits from the ear canal to the eardrum. Then the eardrum vibrates due to the sound wave. After that, the vibration transmits from the eardrum to the three ossicles, and form the three ossicles to the cochlea.

\subsection{Cochlea in Inner Ear}

A cochlea is a part of an inner ear. The cochlea has a snail-like shape and is filled with fluid. There are tiny hair cells as a sensory receptor inside the cochlea. The stapes, namely a part of three osiccles is in contact with the surface of cochlea [15]. In this research, the cochlea was modelized with the translational springs as shown in Figure 1.

\subsection{Ligaments}

An anterior malleal ligament and a lateral malleal one in four ligaments are muscles supporting the malleus in the middle ear as shown in Figure 1. Then a superior malleal ligament and a posterior incudal one in the other two ligaments are muscles supporting the incus in the middle ear as shown in Figure 1. The four ligaments were modelized 


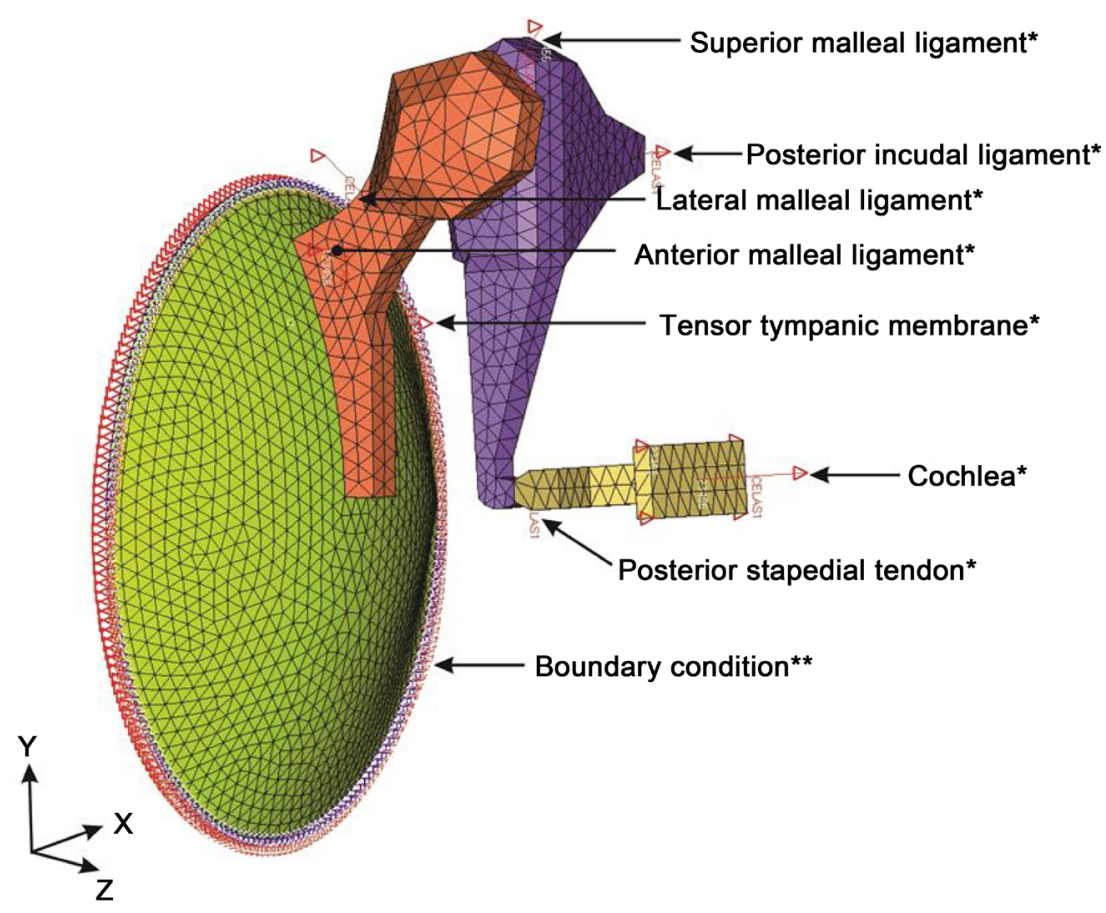

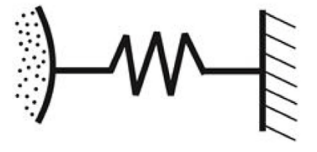

${ }^{*}$ Considered as translational spring

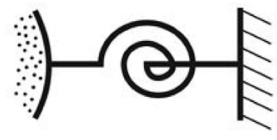

${ }^{* *}$ Considered as torsional spring

Figure 1. Finite element model of total ear system containing a middle ear, a cochlea in an inner ear, four ligaments, a tendon and a tensor tympanic membrane.

with the translational springs in this research. The ends of four ligaments are connected to the wall of middle ear cavity. Then the ends of four translational springs were clamped.

\subsection{Tendon}

A posterior stapedial tendon is a muscle located at the head of the stapes as shown in Figure 1. The stapes is the smallest bone in a human body. The purposes of posterior stapedial tendon are to decrease the vibration of the stapes by pulling on the head of the stapes, to prevent excess motion of the stapes, and to control the amplitude of waves in order to protect the inner ear from the loud sounds. In this research, the tendon was modelized with the translational springs.

\subsection{Tensor Tympanic Membrane}

A tensor tympanic membrane is a muscle attaching to the malleus as shown in Figure 1. The tensor tympanic membrane can regulate the motion of the malleus. If loud sounds are heard, the tensor tympanic membrane reduce the vibration by pulling the malleus away from the eardrum. The tensor tympanic membrane was modelized with 
the translational spring in this research. The end of the tensor tympanic membrane connects to the wall of middle ear cavity. Then the end of translational spring was clamped.

\subsection{Total Ear System}

A total ear system is composed of the middle ear, the cochlea in inner ear, the four ligaments, the tendon and the tensor tympanic membrane as shown in Figure 1. The middle ear consists of an eardrum and three ossicles, namely malleus, incus and stapes. The shapes and dimensions of the eardrum were decided by considering the other researchers [1] [2]. Then, the shapes and dimensions of the three ossicles were decided by considering the references [3] [4]. The CAD software (Solidworks 2015) was used to create the three dimensional model of the middle ear.

In the finite element model of total ear system, the eardrum and the three ossicles were meshed by using six-node triangular elements and ten-node tetrahedron elements, respectively.

\section{Eigen-Value Analysis of Total Ear System}

\subsection{Material Properties of Middle Ear}

Table 1 shows the material properties of middle ear used in the present calculations. The values in Table 1 were decided by considering the references [5] [10].

\subsection{Spring Constants and Boundary Conditions}

Table 2 shows the translational spring constants of ligaments, a tendon, a tensor tym-

Table 1. Material properties of middle ear.

\begin{tabular}{cccc}
\hline Structure & Young's modulus $\left[\times 10^{9} \mathrm{~N} / \mathrm{m}^{2}\right]$ & Mass density $\left[\times 10^{-9} \mathrm{~kg} / \mathrm{m}^{3}\right]$ & Poisson's ratio $[-]^{*}$ \\
\hline Eardrum & 0.033 & 1.20 & 0.3 \\
Malleus & 14.0 & 2.55 & 0.3 \\
Incus & 14.0 & 2.36 & 0.3 \\
Stapes & 14.0 & 2.20 & 0.3 \\
\hline
\end{tabular}

*[-] means a dimensionless quantity.

Table 2. Translational spring constants of ligaments, a tendon, a tensor tympanic membrane and a cochlea.

\begin{tabular}{ccc}
\hline Component & Spring constant, $K_{x}[\mathrm{~N} / \mathrm{mm}]$ & Springconstant, $K_{y}=K_{z}[\mathrm{~N} / \mathrm{mm}]$ \\
\hline Anterior malleal ligament & 1.2 & 0.3 \\
Lateral malleal ligament & 1.2 & 0.3 \\
Superior malleal ligament & 1.2 & 0.3 \\
Posterior incudal ligament & 1.2 & 0.3 \\
Tensor tympanic membrane & 1.2 & 0.3 \\
Posterior stapedial tendon & 1.2 & 0.3 \\
Cochlea & 0.2 & - \\
\hline
\end{tabular}


panic membrane and a cochlea. As for the ligaments, the tendon and the tensor tympanic membrane, each of them was considered as three translational springs in $x, y$ and $z$ directions. The indexes, $x, y$ and $z$ of each spring constant denote the local coordinates of itself. The $x$-direction of each local coordinate frame was defined in the normal direction to the surface of an ossicle. The values of translational spring constants, $K_{i}(i$ $=x, y, z)$ for the ligaments, the tendon, the tensor tympanic membrane and the cochlea were decided by trial and error so that the stapes can perform a piston motion in the $x$-direction of local coordinate frame. The stapes contacting with the cochlea moves like a piston in the $x$-direction.

As for the boundary conditions of the eardrum, a local coordinate frame was defined at each node of boundary of the eardrum. In each the local coordinate frame, three translational motions in the $x$-, $y$ - and $z$-directions and two rotational ones around the $x$-, and $z$-axes were clamped. Then, the torsional springs of $K_{\theta y}$ were applied to the rotational motions around the $y$-axes, on the nodes of boundary of the eardrum. The torsional springs of $K_{\theta y}$ were used to adjust the stiffness of boundary of the eardrum in order to make the boundary conditions of the eardrum similar to those of a human eardrum. As for the torsional springs on boundary of the eardrum, the same values, $K_{\theta_{y}}=$ $3.0 \times 10^{-5}[\mathrm{Nmm} / \mathrm{rad}]$ as the authors' previous report [15] were used.

\subsection{Calculation Method}

The Optistruct of Hypermesh was used to carry out eigen-value analysis of total earsystem. The eigen-value analysis of the total ear system was carried out to obtain the natural frequencies and the vibration modes in the frequency range from $100[\mathrm{~Hz}]$ to $10,000[\mathrm{~Hz}]$.

\subsection{Results}

Figure 2 shows the vibration modes of the total ear system. The first, second and third vibration modes have similar modes, but the directions of motions of the three ossicles are different. Then the first vibration mode has one small loop. The three ossicles move in the normal direction to the surface of the eardrum in $f_{1}=1033[\mathrm{~Hz}]$. The second vibration mode in $f_{2}=1728[\mathrm{~Hz}]$ has two small loops. The directions of motions of the three ossciles in $f_{2}=1728[\mathrm{~Hz}]$ are different from the first one in $f_{1}=1033[\mathrm{~Hz}]$. Furthermore, the third vibration mode in $f_{3}=1846[\mathrm{~Hz}]$ has two small loops, and the directions of motions of the three ossicles are different. The fourth, fifth and sixth vibration modes have similar modes in which the displacements at loops of the three ossicles are very small. The fourth vibration mode in $f_{4}=2659$ [Hz] has two loops in which the both loops have the same direction on displacements. The fifth vibration mode in $f_{5}=2697[\mathrm{~Hz}]$ also has two loops, but one loop moves in the opposite direction to the other one. Then, the sixth vibration mode has four large loops and one small loop in $f_{6}=3013[\mathrm{~Hz}]$.

\section{Frequency Response Analysis of Total Ear System}

\subsection{Calculation Method}

Figure 3 shows the structural dampings used for the total ear system using the struc- 


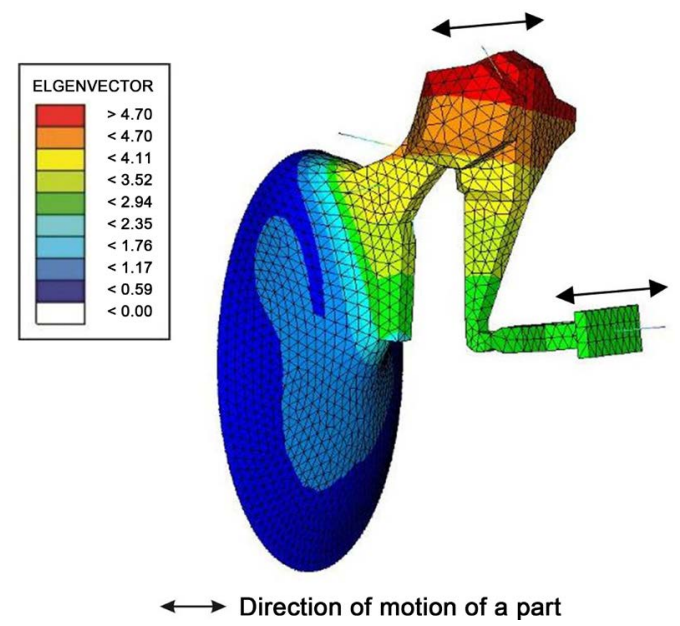

(a)

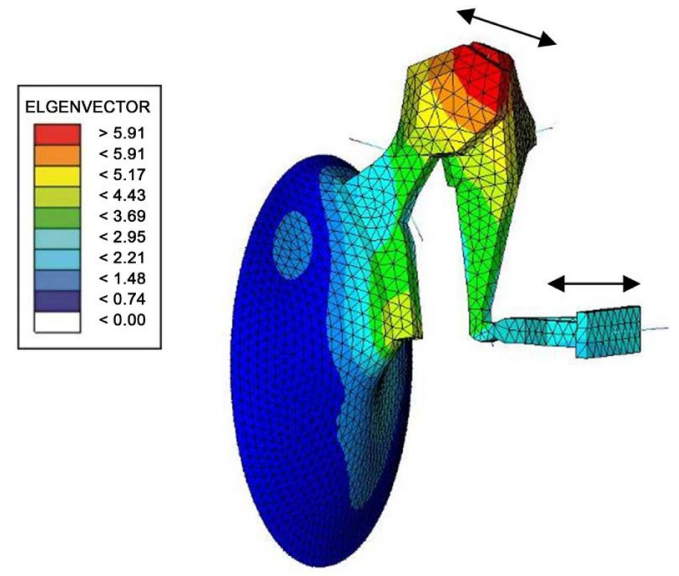

$\longleftrightarrow$ Direction of motion of a part

(c)

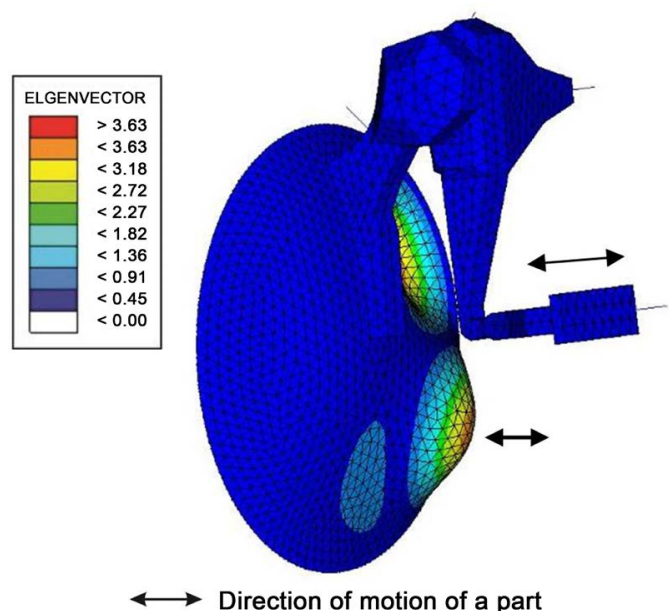

(e)

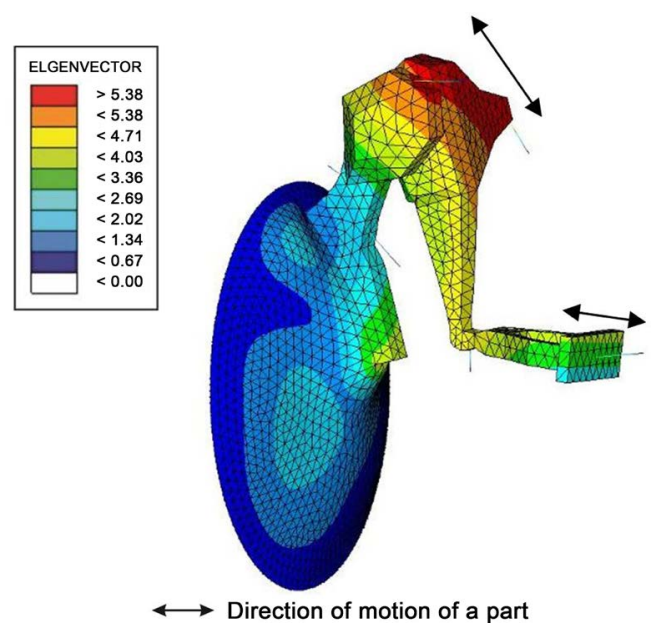

(b)

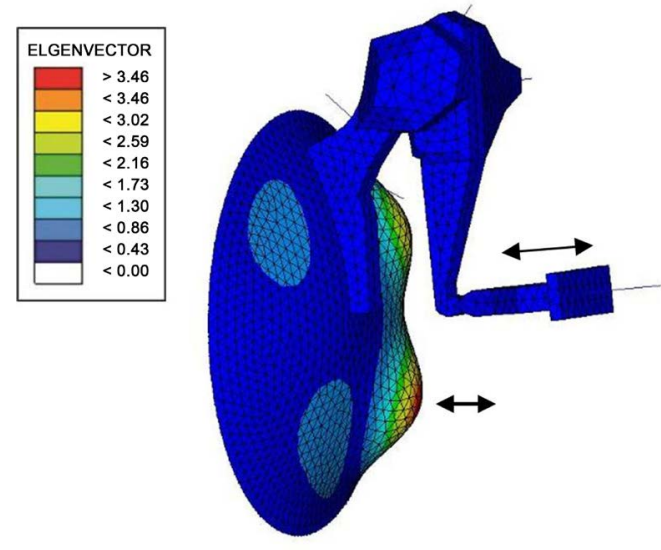

Direction of motion of a part

(d)

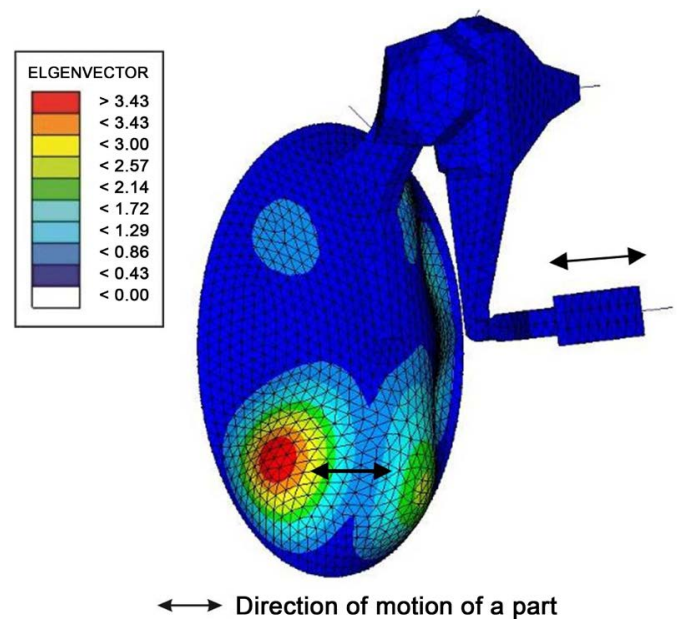

(f)

Figure 2. Vibration modes of the total ear system. (a) Vibration mode of 1st natural frequency, $f_{1}=1033$ [Hz]; (b) Vibration mode of 2nd natural frequency, $f_{2}=1728[\mathrm{~Hz}]$; (c) Vibration mode of 3rd natural frequency, $f_{3}=1846[\mathrm{~Hz}]$; (d) Vibration mode of 4th natural frequency, $f_{4}=2659[\mathrm{~Hz}] ;$ (e) Vibration mode of 5th natural frequency, $f_{5}=2697[\mathrm{~Hz}]$; (f) Vibration mode of 6th natural frequency, $f_{6}=3013$ $[\mathrm{Hz}]$. 


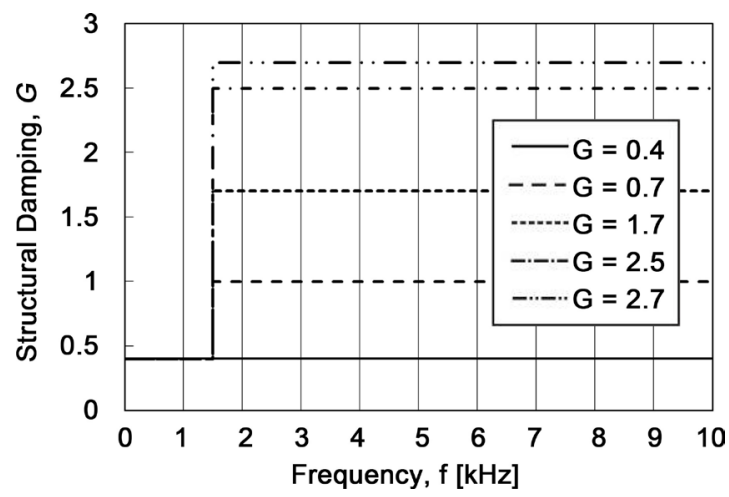

Figure 3. Structural dampings used for the total ear system using structural damping coefficient of $G=0.4$ in $1500[\mathrm{~Hz}]$ and less and five kinds of values on $G$ in $1500[\mathrm{~Hz}]$ and more.

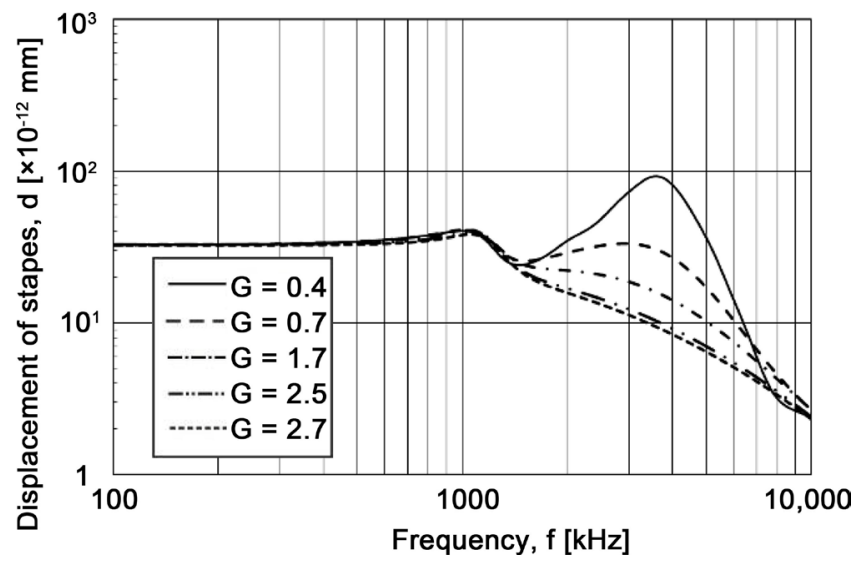

Figure 4. Frequency response of the stapes displacement of the total ear system.

tural damping coefficient of $G=0.4$ in $1500[\mathrm{~Hz}]$ and less, and five kinds of values on $G$ in $1500[\mathrm{~Hz}]$ and more. The Optistruct of Hypermesh was used to carry out the frequency response analyses of the total ear system using the torsional spring constant, $K_{\theta y}$ $=3.0 \times 10^{-5}[\mathrm{Nmm} / \mathrm{rad}]$ on the boundary under the sound pressure level, $P=2.0 \times 10^{-6}$ $[\mathrm{Pa}]$ in the frequency range from $100[\mathrm{~Hz}]$ to $10,000[\mathrm{~Hz}]$. Then five structural damping coefficients were used for the frequency response analyses to examine the effect of structural damping coefficients on the displacements of stapes in $1500[\mathrm{~Hz}]$ and more.

\subsection{Results}

Figure 4 shows the frequency responses of displacements of the stapes of the total ear system. The use of structural damping coefficient of $G=0.4$ can make the displacement constant at the frequency less than $1000[\mathrm{~Hz}]$. However, the value of $G=0.4$ makes the displacement very large in $1500[\mathrm{~Hz}]$ and more. Therefore, the frequency response analyses using five kinds of structural damping coefficients were carried out to decrease the large displacements in $1500[\mathrm{~Hz}]$ and more. Then, the value of $G=2.5$ was selected as the structural damping coefficient in $1500[\mathrm{~Hz}]$ and more. Furthermore, it can be seen that the first natural frequency of the total ear system became around $1000[\mathrm{~Hz}]$. It is well known that the first natural frequency of a human middle ear becomes around 
$1000[\mathrm{~Hz}][7][10]$. Then, the frequency responses calculated in this study show the similar results reported by the other researchers [7] [9].

\section{Time History Response Analysis of Total Ear System}

The FEA by the Hypermesh was used to carry out time history response analysis of total ear system. In this analysis, the human voices created as input sound pressure were used as the external forces.

\subsection{Creating Input Sound Pressures}

Figure 5 shows the time histories of original sound pressures downloaded from the website opening samples umanvoices as wav files

(http://www.geocities.jp/onsei2007/wav_data51/wav_data51.html). Firstly, the downloaded human voices such as " $\vec{i}$ ", " $u$ " and " $e$ " were converted to csv files using Scilab. Each human voice has a single channel, namely a mono sound in 0.25 [s].

Then two types of sounds were created by using the downloaded original sound pressures. The first type of sounds are the sound pressures in 0.022 [s] to use as input data, namely external forces in Hypermesh. The second type of them are the sound pressures in $2[s]$ to evaluate the sounds by listening them. Figure 6 shows the time histories of sound pressures in 0.02 [s] created by connecting three basic waveforms so

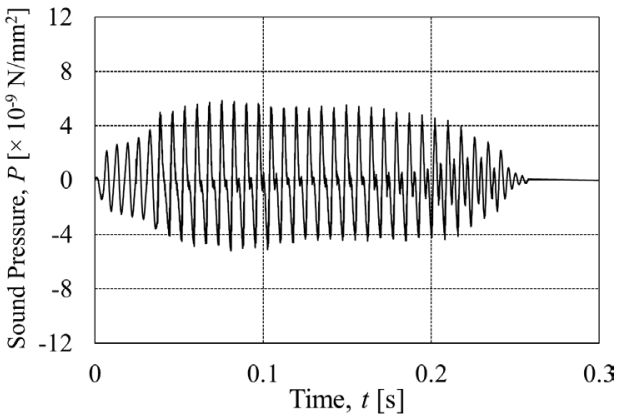

(a)

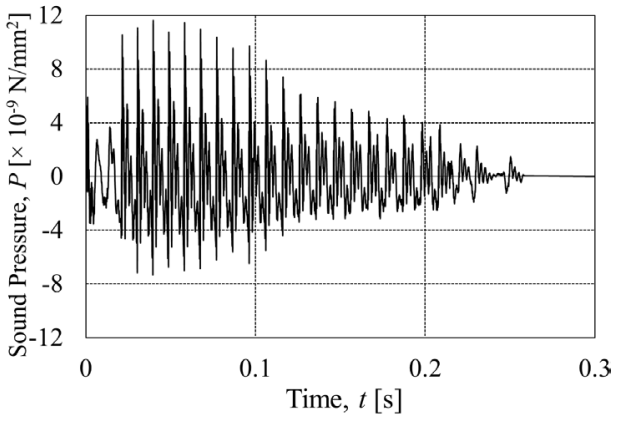

(b)

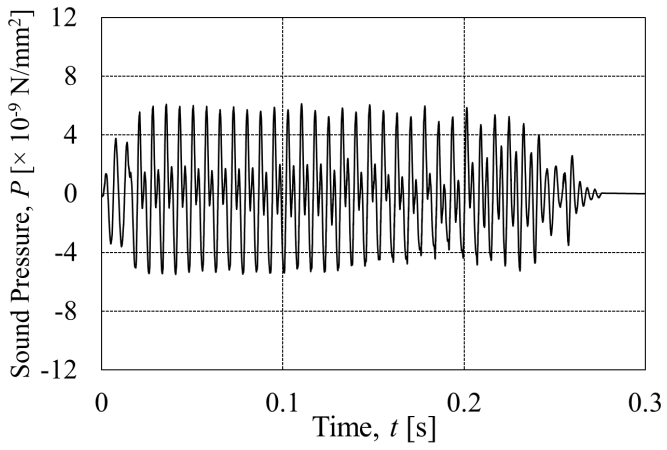

(c)

Figure 5. Time histories of original sound pressures downloaded from a website opening samples of humanvoices as wav files(http://www.geocities.jp/onsei2007/wav_data51/wav_data51.html). (a) Original sound pressure on vowel " 1 "; (b) Original sound pressure on vowel " $u$ "; (c) Original sound pressure on vowel "e". 


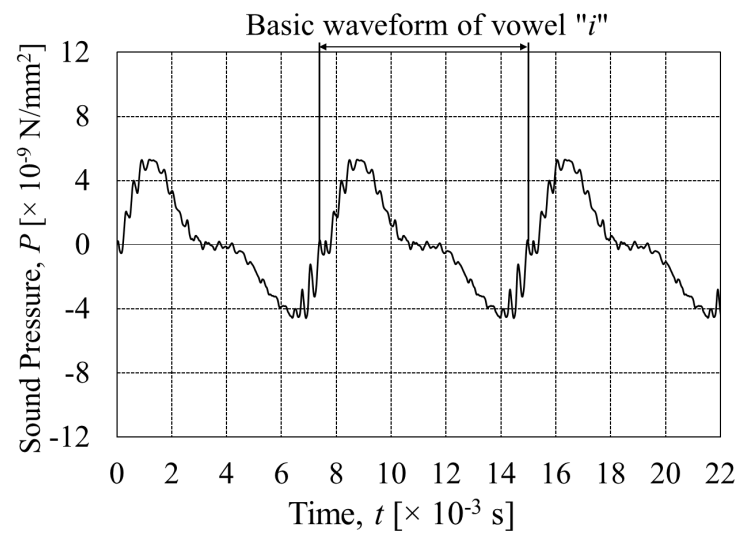

(a)

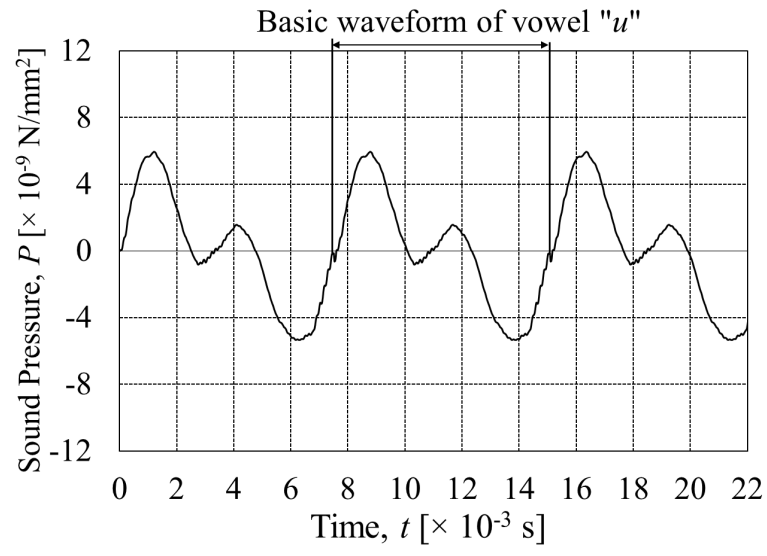

(b)

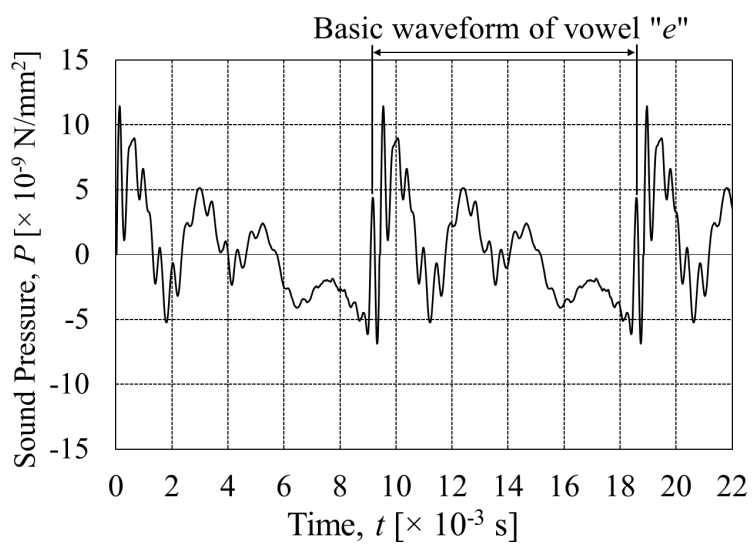

(c)

Figure 6. Time histories of sound pressures in 0.022 [s] created by connecting three basic waveforms so that they may not exceed the limitation of input data in Hypermesh. (a) Created sound

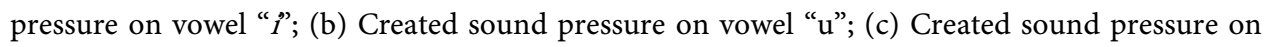
vowel " $e$ ".

that they may not exceed the limitation of input data in Hypermesh. As for the second type of sounds, they were created as data in $2[\mathrm{~s}]$ so that the authors can evaluate them, by the same method as the first type of them. The waveforms of created sound pres- 
sures on vowel " $e$ " are a little more complex than vowels " $i$ " and " $u$ ".

Figure 7 shows the evaluation of differences among the original sounds, the sounds in 2 [s] created as input data of Hypermesh and the sounds in 2 [s] created by using the results calculated by Hypermesh. The created sound pressures of csv files were converted to wav files by using Scilab. Then, the original sounds and the sounds in 2 [s] created in the same way as input data of Hypermesh of wav files were played in order to evaluate them before using them as input data of Hypermesh. Then, the original sounds and the created sounds were compared for each vowel. The results of evalutions showed that the created sounds on vowels "i", "u" and "e" were identical to its original sounds. Therefore, the created sounds as input data for the three vowels were feasible to input them into the Hypermesh in order to carry out the time history response analysis of the total ear system.

\subsection{Calculation Method}

The Optistruct in HyperWorks was used to carry out time history responses analysis of total ear system. The sound pressure in 0.022 [s] on vowel " $\vec{i}$ ", " $u$ " or " $e$ " created for input data was loaded to the eardrum of total ear system as the external force.

Then, as for the structural damping coefficients, $G$, the values of $G=0.4$ and $G=2.5$ were used in $1500[\mathrm{~Hz}]$ and less, and in $1500[\mathrm{~Hz}]$ and more, respectively. As for the incremental time, $\Delta t$ and the number of time, $N, \Delta t=1.0 \times 10^{-4}[\mathrm{~s}]$ and $N=220$ were used, respectively.

\subsection{Results}

Figure 8 shows the calculated time history responses of displacements at the stapes when the eardrum was subjected to the created sound pressures. In comparison between the created input data shown in Figure 7 and the calculated output ones shown in Figure 8, it is understood that high frequency components of the calculated output data reduce due to an effect of the structural damping.

The sounds in 2 [s] created by using the calculated output data of csv files were converted to wav files by using Scilab. Then both the sounds in $2[\mathrm{~s}]$ created in the same way as the created input data and the sounds in 2 [s] created by using the calculated output ones of wav files were played in order to evaluate them. The evaluation of differences between both the sounds in 2 [s] was carried out as shown in Figure 7. In the
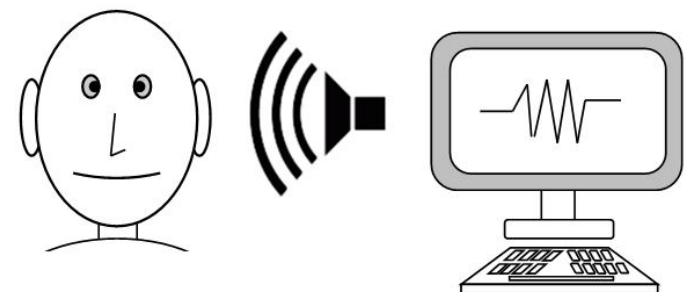

Figure 7. Evaluation of differences among the original sounds, the sounds in 2 [s] created as input data of Hypermesh and the sounds in 2 [s] created by using the results calculated by Hypermesh. 


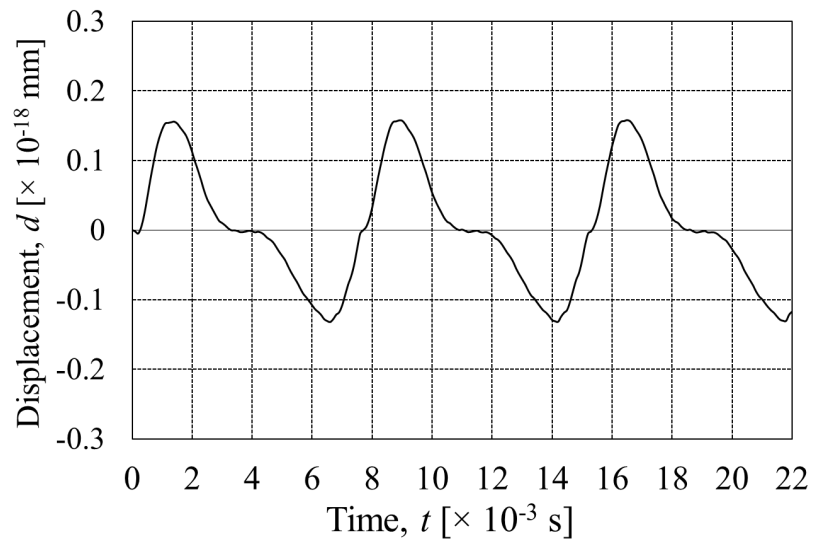

(a)

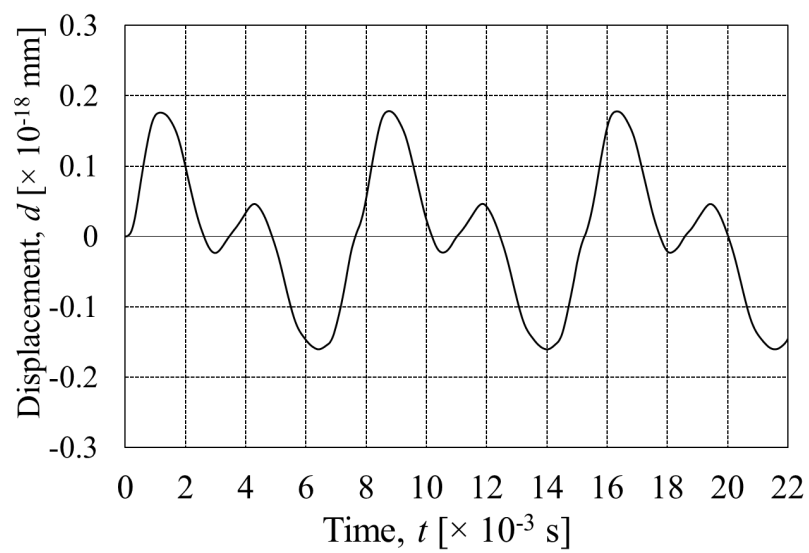

(b)

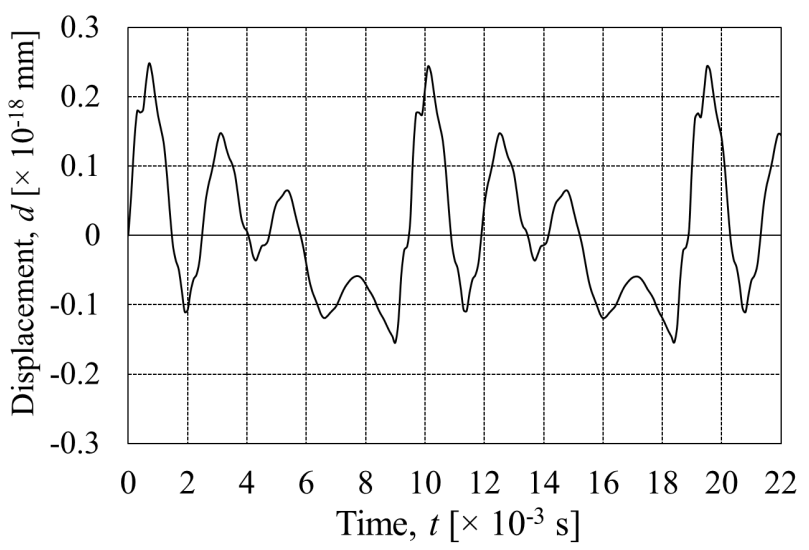

(c)

Figure 8. Calculated time history responses of displacements at the stapes when the eardrum was subjected to the created sound pressures. (a) Displacement for vowel " $i$;; (b) Displacement for vowel " $u$ "; (c) Displacement for vowel " $e$ ".

results of evaluation, it was obtained that the sounds of calculated output data could be heard more clearly than those of the created input ones because the high frequency components of calculated output data reduced due to the effect of the structural damping. 


\section{Conclusions}

In the present research, the simple model proposed by the authors was evaluated by dynamic analyses such as eigen-value, frequency response and time history response analyses. As for the time history response analysis, the current research was performed using the human voices as the external forces. The summary of the results is shown below.

1) The eigen-value analysis had been carried out to obtain natural frequencies and vibration modes of the total ear system using the torsional springs on the boundary of the eardrum and the translational springs for ligaments, a tensor tympanic membrane, a tendon and a cochlea.

2) It was determined that the structural damping coefficient, Gis 0.4 for less than $1500[\mathrm{~Hz}]$ and 2.5 for more than $1500[\mathrm{~Hz}]$ by the frequency response analysis of the total ear system.

3) It was obtained that the sounds of calculated output data could be heard more clearly than those of the created input ones because the high frequency components of calculated output data reduced due to the effect of the structural damping.

In order to improve the simple model, the variation of external forces in the time history response analysis of the total ear system such as created sound pressures on consonants will be considered in the future.

\section{Acknowledgements}

The authors would like to thank Altair Engineering, Ltd. for providing the license of Hyper Works by Academic Open Program (AOP).

\section{References}

[1] Wada, H., Kobayashi, T., Naganuma, H. and Tachizaki, H. (1990) Analysis of Dynamic Characteristics of Eardrum (Young's Modulus, Thickness and Damping Ratio of Human Eardrum). Japan Society of Mechanical Engineers, 56, 87-90.

[2] Funnel, W.R.J. and Lazlo, C.A. (1978) Modelling of the Cat Eardrum as a Thin Shell Using Finite Element Method. Journal of the Acoustical Society of America, 63, 1461-1467. https://doi.org/10.1121/1.381892

[3] De Graf, D., Aernouts, J., Aerts, J., Cheng, J.T., Horwitz, R., Rosowski, J.J. and Dirckx, J.J. (2014) Viscoelatic Properties of the Human Tympanic Membrane Studied with Stroboscopic Holography. Hearing Research Journal, 312, 69-80. https://doi.org/10.1016/j.heares.2014.03.002

[4] Bohnke, F., Bretan, T., Lehner, S. and Strenger, T. (2013) Simulations and Measurements of Human Middle Ear Vibrations Using Multi-Body Systems and Laser Doppler Vibrometry with the Floating Mass Tranducer. Journal of Materials, 6, 4675-4688. https://doi.org/10.3390/ma6104675

[5] Liu, Y., Li, S. and Sun, X. (2009) Numerical Analysis of Ossicular Chain Lesion of Human Ear. Acta Mechanica Sinica, 25, 241-247. https://doi.org/10.1007/s10409-008-0206-6

[6] Skordzka, E. and Modlawska, J. (2006) Modal Analysis of the Human Tympanic Membrane of Middle Ear Using the Finite-Element Method. Achieves of Acoustics, 31, 23-28.

[7] Lee, C.-F., Chen, P.R., Lee, W.J., Chen, J.H. and Liu, T.C. (2006) Computer Aided Three 
Dimensional Reconstruction and Modelling of Middle Ear Biomechanics by High-Resolution Computed Tomography and Finite Element Analysis. Biomedical Engineering, Basis \& Communications, 188, 214-221.

[8] Gan, R.Z., Feng, B. and Sun, Q. (2004) Three-Dimensional Finite Element Modelling of Human Ear for Sound Transmission. Annals of Biomedical Engineering, 32, 847-859. https://doi.org/10.1023/B:ABME.0000030260.22737.53

[9] Sun, Q., Chang, K.H., Dormer, K.J., Dyer Jr., R.K. and Gan, R.Z. (2002) An Advanced Computer-Aided Geometric Modeling and Fabrication for Human Middle Ear. Medical Engineering \& Physics, 2, 595-606. https://doi.org/10.1016/S1350-4533(02)00045-0

[10] Koike, T. and Wada, H. (2002) Modelling of the Human Middle Ear Using the Finite Element Method. Journal of the Acoustical Society of America, 43-64.

[11] Tae-Soo, A., et al. (2013) Experimental Measurement of Tympanic Membrane Response for Finite Element Model Validation of a Human Middle Ear. Springer Plus, 2, 527. https://doi.org/10.1186/2193-1801-2-527

[12] Ihrle, S., Lauxmann, M., Eiber, A. and Eberhar, P. (2012) Nonlinear Modelling of the Middle Ear as an Elastic Multibody System-Applying Model Order Reduction to AcoustoStructural Coupled System. Journal of Computational and Applied Mathematics, 18-26.

[13] Homma, K., Du, Y., Shimizu, Y. and Puria, S. (2008) Ossicular Resonance Modes of the Human Middle Ear for Bone and Air Conduction. Journal of the Acoustical Society of America, 968-979.

[14] Hidayat, Okamoto, S., Lee, J.H., Matsuura, K., Hato, N., Yamada, H. and Takagi, D. (2016) Dynamics Analyses of Human Middle Ear System Using Finite Element Method. Full Paper Proceeding ECBA-2016, 127, 10-21.

[15] Moller, A.R. (2006) Hearing: Anatomy, Physiologi, and Disorders of the Auditory System. 2nd Edition, Academic Press.

Submit or recommend next manuscript to SCIRP and we will provide best service for you:

Accepting pre-submission inquiries through Email, Facebook, LinkedIn, Twitter, etc.

A wide selection of journals (inclusive of 9 subjects, more than 200 journals)

Providing 24-hour high-quality service

User-friendly online submission system

Fair and swift peer-review system

Efficient typesetting and proofreading procedure

Display of the result of downloads and visits, as well as the number of cited articles

Maximum dissemination of your research work

Submit your manuscript at: http://papersubmission.scirp.org/

Or contact jbise@scirp.org 